PROCEEDINGS OF THE

AMERICAN MATHEMATICAL SOCIETY

Volume 125, Number 2, February 1997, Pages 507-514

S 0002-9939(97)03688-5

\title{
CHERN CHARACTERS ASSOCIATED WITH ALMOST COMMUTING ALGEBRAS
}

\author{
DAOXING XIA
}

(Communicated by Palle E. T. Jorgensen)

\begin{abstract}
A trace formula related to $p$-almost commuting subalgebras $X$ and $Y$ is established. By means of this formula, homomorphisms from $K_{0}(X)$ to $H_{\lambda}^{\text {odd }}(Y)$ and from $K_{1}(X)$ to $H_{\lambda}^{\text {even }}(Y)$ are established. An index map from $K_{0}(X) \times K_{1}(Y)$ to $\mathbb{Z}$ is also given.
\end{abstract}

\section{INTRODUCTION}

Let $\mathcal{A}$ be an algebra over $\mathbb{C}$ with trace ideal $J$ and trace $\tau$. Assume $X$ and $Y$ are two subalgebras of $\mathcal{A}$ satisfying the condition that there is a natural number $p$ such that

$$
\left[x^{1}, y^{1}\right] \cdots\left[x^{p}, y^{p}\right] \in J
$$

for $x^{j} \in X$ and $y^{j} \in Y$, where $[x, y]$ is the commutator $x y-y x$. Then we say that $X$ and $Y$ are p-almost commuting. The present note is a continuation of [6], [7], [8], [9] to study the Chern characters associated with this pair of $X$ and $Y$ in the context of A. Connes' theory of noncommutative geometry. First in $\S 2$, we establish a trace formula (8) of

$$
\xi_{n}\left(x^{0}, \ldots, x^{n} ; y^{0}, \ldots, y^{n-1}\right) \stackrel{\text { def }}{=} \tau x^{0}\left[x^{1}, y^{0}\right] \cdots\left[x^{n}, y^{n}\right], \quad n \geq p,
$$

which gives the relation of the cyclic cochains $A_{x} A_{y} \xi_{k}$ and $A_{x} A_{y} \xi_{k+2 m}$ in terms of cyclic cohomology (see Theorem 1). As a corollary, it reduces to the trace formula of

$$
\varphi_{n}\left(x^{0}, \ldots, x^{n} ; y^{0}, \ldots, y^{n}\right)=\tau\left[x^{0}, y^{0}\right] \cdots\left[x^{n}, y^{n}\right], \quad n \geq p-1,
$$

in $[7]$ and $[8]$.

Based on Theorem 1 and A. Connes' theory, in Theorem 2 and Theorem 3 we establish homomorphisms from $K_{0}(X)$ to $H_{\lambda}^{\text {odd }}(Y)$ and $K_{1}(X)$ to $H_{\lambda}^{\text {even }}(Y)$ by means of Chern characters. But for convenience, in the statement of Theorem 3, we switch the roles of $X$ and $Y$. In Theorem 4, we give the index map from $K_{0}(X) \times K_{1}(Y)$ to $\mathbb{Z}$ in the case when $\mathcal{A}$ is an algebra of operators on a separable complex Hilbert space. We also give a simple example to show that the index map is not trivial.

Received by the editors August 16, 1995.

1991 Mathematics Subject Classification. Primary 47A55; Secondary 47G05.

Key words and phrases. Cyclic cohomology, Chern character, K-theory, almost commuting, deformed commutator, twisted commutator.

This work is supported in part by NSF grant DMS-9400766.

(C)1997 American Mathematical Society 
In $\S 4$, we generalize Theorem 1 to the $q$-deformed (or $q$-twisted) commutator case (see Theorem $1^{\prime}$ ). Besides, we give a theorem (Theorem 5 ) of Chern character of odd dimension which is just a remark of the previous work in [9].

\section{BASIC FORMULAS}

Let $X$ be an algebra over $\mathbb{C}$. Let $C^{n}=C^{n}(X)$ be the space of multilinear functions $f_{n}\left(x^{0}, \ldots, x^{n}\right), x^{j} \in X$, let $t: C^{n} \rightarrow C^{n}$ be the operation

$$
\left(t f_{n}\right)\left(x^{0}, \ldots, x^{n}\right)=(-1)^{n} f_{n}\left(x^{n}, x^{0}, \ldots, x^{n-1}\right),
$$

let $C_{\lambda}^{n}=\left\{f \in C^{n}: t f=f\right\}$, and let $A f=\left(1+t+\cdots+t^{n}\right) f, f \in C^{n}$. Let $b$ be the Hochshild operation $C^{n} \rightarrow C^{n+1}$,

$$
(b f)\left(x^{0}, \ldots, x^{n+1}\right)=\left(b^{\prime} f\right)\left(x^{0}, \ldots, x^{n+1}\right)+(-1)^{n+1} f\left(x^{n+1} x^{0}, \ldots, x^{n}\right),
$$

where $\left(b^{\prime} f\right)\left(x^{0}, \ldots, x^{n+1}\right)=\sum_{j=0}^{n}(-1)^{j} f_{n}\left(x^{0}, \ldots, x^{j} x^{j+1}, \ldots, x^{n}\right)$. Let $p$ be the operation $p f=\sum_{j=0}^{n}(n-j) t^{j} f, f \in C^{n}$, where $t^{0}=1$. Define $S=2 \pi i b p b^{\prime}$. Then this operator $S$ coincides with A. Connes' operator [1] $S$ at $Z_{\lambda}^{n}=\left\{f \in C_{\lambda}^{n}: b f=0\right\}$. Let $q$ be the operation [8] $q f=n A f / 2-p t f, f \in C^{n}$. Let $r$ be the operation [8] $r f=r_{n}(t) f, f \in C^{n}$, for $n>0$, where $r_{n}(\cdot)$ is a polynomial of degree $\leq n-1$ satisfying $q f=(1-t) r f, f \in C^{n}$. For convenience, define $\hat{S}=b p b^{\prime}$ (which is the operator $S$ in [8] and [9]).

The following well-known identities will be needed. Notice that $(1-t) b=b^{\prime}(1-t)$ (cf. [4], [1]) and that $p(1-t) f=(n+1-A) f$, for $f \in C^{n}$ (cf. [8]). Hence

$$
\hat{S}(1-t)=-b A b
$$

and

$$
(1-t) \hat{S}=-b^{\prime} b A .
$$

If $f \in C_{\lambda}^{n}$, then $S b f \in C_{\lambda}^{n+3}$ and (cf. [8])

$$
\hat{S} b f=\frac{1}{n+1} b A \hat{S} f, \quad f \in C_{\lambda}^{n} .
$$

In the present note, the tensor product spaces $C^{n}(X) \otimes C^{n}(Y)$ and $C_{\lambda}^{n}(X) \otimes$ $C_{\lambda}^{n}(Y)$ of functions of several variables $x^{i} \in X$ and $y^{j} \in Y$ are considered. The operations $t_{x}, b_{x}, b_{x}^{\prime}, p_{x}, \ldots$ or $t_{y}, b_{y}, b_{y}^{\prime}, p_{y}, \ldots$ are the corresponding operations $t, b, b^{\prime}, p, \ldots$ with respect to variables $x$ 's and $y$ 's respectively.

In $\S 2$ and $\S 3$, assume that $X$ and $Y$ are two $p$-almost commuting subalgebras of an algebra $\mathcal{A}$ over $\mathbb{C}$ with trace ideal $J$ and trace $\tau$. Define for $n \geq p$

$$
\begin{aligned}
\psi_{n}\left(x^{0}, \ldots, x^{n} ; y^{0}, \ldots, y^{n}\right) & =\tau x^{0} y^{0}\left[x^{1}, y^{1}\right] \cdots\left[x^{n}, y^{n}\right], \\
\phi_{n-1}\left(x^{0}, \ldots, x^{n-1} ; y^{0}, \ldots, y^{n-1}\right) & =\tau\left[x^{0}, y^{0}\right] \cdots\left[x^{n-1}, y^{n-1}\right], \\
\xi_{n}\left(x^{0}, \ldots, x^{n} ; y^{0}, \ldots, y^{n-1}\right) & =\tau x^{0}\left[x^{1}, y^{0}\right] \cdots\left[x^{n}, y^{n-1}\right], \\
\eta_{n}\left(x^{0}, \ldots, x^{n-1} ; y^{0}, \ldots, y^{n}\right) & =\tau y^{0}\left[x^{0}, y^{1}\right] \cdots\left[x^{n-1}, y^{n}\right] .
\end{aligned}
$$

The following is a special case of Lemma 1 in [9] (or [7], [8]). 
Lemma 1. For $n \geq p$,

$$
\begin{aligned}
\xi_{n+1}=b_{x} \psi_{n}, & \eta_{n+1}=-t_{x} b_{y} \psi_{n}, \\
\left(1-t_{x}\right) \xi_{n}=b_{x}^{\prime} \phi_{n-1}, & \left(1-t_{y}\right) \eta_{n}=b_{y}^{\prime} \phi_{n-1}, \\
\left(1-t_{y}^{-1}\right) \xi_{n}=b_{x} \phi_{n-1}, & \left(1-t_{x}^{-1}\right) \eta_{n}=b_{y} \phi_{n-1}, \\
\left(1-t_{y}\right) \psi_{n}=b_{y}^{\prime} \xi_{n}-t_{y} \phi_{n}, & \left(1-t_{x}\right) \psi_{n}=b_{x}^{\prime} \eta_{n}+\phi_{n} .
\end{aligned}
$$

Theorem 1. Let $k \geq p$ be an even number, $m$ be a natural number, and $n=k+2 m$. Then there are $\theta_{j} \in C_{\lambda}^{j}(X) \otimes C_{\lambda}^{j}(Y), j=n-1, n-2\left(\theta_{j}\right.$ also depending on $\left.k\right)$, such that

$$
A_{x} A_{y} \xi_{n}=b_{x} \theta_{n-1}+A_{x} S_{x} b_{y} \theta_{n-2}+\tilde{\xi}_{n}
$$

where

$$
\tilde{\xi}_{n}=\frac{1}{(2 \pi)^{2 m}} \frac{k !(k-1) !}{n !(n-1) !}\left(A_{x} S_{x}\right)^{m}\left(A_{y} S_{y}\right)^{m} A_{x} A_{y} \xi_{k} .
$$

Proof. Based on Lemma 1, in [8] (cf. (20) of [8]) it is proved that

$$
A_{x} A_{y} \psi_{j}=(j+1)^{2} \psi_{j}-(j+1) p_{y} b_{y}^{\prime} \xi_{j}-p_{x} b_{x}^{\prime} A_{y} \eta_{j}-(j+1) q_{y} \phi_{j},
$$

for $j \geq p$. From (4), (10) and the fact that $b_{x} \xi_{j}=0$, we have

$$
\xi_{j+1}=\frac{1}{(j+1)^{2}}\left(b_{x} A_{x} A_{y} \psi_{j}+\hat{S}_{x} A_{y} \eta_{j}\right)+\frac{1}{j+1} b_{x} q_{y} \phi_{j},
$$

for $j \geq p$. Thus

$$
A_{x} A_{y} \xi_{j+1}=\frac{j+2}{j+1} b_{x} A_{x} A_{y} \psi_{j}+\frac{1}{j+1} A_{x} \hat{S}_{x} A_{y} \eta_{j},
$$

for $j \geq p$, since $A_{y} q_{y}=0$.

Similarly, we have

$$
\eta_{j+1}=-\frac{1}{(j+1)^{2}}\left(A_{x} b_{y} A_{y} \psi_{j}+A_{x} \hat{S}_{y} \xi_{j}\right)-\frac{1}{j+1} t_{x} b_{y} q_{y} \phi_{j},
$$

for $j \geq p$. It is easy to see that $r_{x} \phi_{j}=r_{y} \phi_{j}$, since $t_{x} t_{y} \phi_{j}=\phi_{j}$. Denote $r_{x} \phi_{j}$ by $r \phi_{j}$ (cf. [8]). From (1), we have

$$
\begin{aligned}
\hat{S}_{x} t_{x} q_{y} \phi_{j} & =\hat{S}_{x} t_{x}\left(1-t_{y}\right) r_{y} \phi_{j}=-\hat{S}_{x}\left(1-t_{x}\right) r_{y} \phi_{j} \\
& =b_{x} A_{x} b_{x} r \phi_{j}
\end{aligned}
$$

for $j \geq p-1$. From (6), it is easy to see that $t_{y}^{l} \xi_{j}=\xi_{j}+\sum_{v=1}^{l} t_{y}^{v} b_{x} \phi_{j-1}$. Thus

$$
A_{y} \xi_{j}=j \xi_{j}+b_{x} p_{y} t_{y} \phi_{j-1}, \quad j \geq p .
$$

Similar to (14), we have $\hat{S}_{y} q_{y} \phi_{j}=-b_{y} A_{y} b_{y} r \phi_{j}$. From (7), $A_{y} \phi_{j}=b_{y} A_{y} \xi_{j}$. Hence $b_{x} A_{y} \phi_{j}=0$. Thus

$$
b_{x} \hat{S}_{y} t_{y} p_{y} \phi_{j}=b_{x} \hat{S}_{y}\left(j A_{y} \phi_{j} / 2-q_{y} \phi_{j}\right)=b_{x} b_{y} A_{y} b_{y} r \phi_{j} .
$$


From (12), (13), (14), (15) and (16), we have, for $j \geq p$,

$$
\begin{aligned}
A_{x} A_{y} \xi_{j+2}= & \frac{j+3}{j+2} b_{x} A_{x} A_{y}\left(\psi_{j+1}-\frac{1}{j+1} b_{x} b_{y} r \phi_{j}\right) \\
& -\frac{1}{(j+1)^{2}} A_{x} \hat{S}_{x} A_{x} b_{y} A_{y}\left(\psi_{j}-\frac{1}{j} b_{x} b_{y} r \phi_{j-1}\right) \\
& -\frac{1}{(j+2)(j+1)^{2} j} A_{x} \hat{S}_{x} A_{x} A_{y} \hat{S}_{y} A_{y} \xi_{j} .
\end{aligned}
$$

As in [8], define $\Theta_{k-1}=\Theta_{k-2}=0$,

$$
\zeta_{j}=\psi_{j}-\frac{1}{j} b_{x} b_{y} r \phi_{j-1}-\frac{1}{j(j-1)^{2}} \hat{S}_{x} \hat{S}_{y} \Theta_{j-2}
$$

and $\Theta_{j}=\frac{1}{j+1} A_{x} A_{y} \zeta_{j}$ for $j \geq k$ by mathematical induction. Define

$$
\hat{\xi}_{j}=A_{x} A_{y} \xi_{j}-(j+1) b_{x} \Theta_{j-1}+\frac{1}{j-1} A_{x} \hat{S}_{x} b_{y} \Theta_{j-2} .
$$

From (3) and (17), we have

$$
\hat{\xi}_{j+2}=-A_{x} \hat{S}_{x} A_{y} \hat{S}_{y} \hat{\xi}_{j} /(j+2)(j+1)^{2} j, \quad j \geq k,
$$

which proves (8) and (9) where $\theta_{n-1}=(n+1) \Theta_{n-1}$ and $\theta_{n-2}=-\Theta_{n-2} /(n-1) 2 \pi i$.

Since $A_{x} \phi_{n}=A_{y} \phi_{n}$, denote it by $A \phi_{n}$. Applying $b_{y}$ to (8), we get the following.

Corollary 1 [8]. Let $k \geq p$ be an even number, $m$ be a natural number and $n=$ $k+2 m$. Then there is $\Theta_{n-1} \in C_{\lambda}^{n-1}(X) \otimes C_{\lambda}^{n-1}(Y)$ such that

$$
A \phi_{n}=b_{x} b_{y} \Theta_{n-1}+\tilde{\phi}_{n},
$$

where $\tilde{\phi}_{n}=k ! S_{x}^{m} S_{y}^{m} A \phi_{n} /(2 \pi)^{2 m} n !$.

\section{Chern characters}

Let $\ell \geq 1$. For $\left(x_{i j}\right) \in M_{\ell}(X)$, let $\left[\left(x_{i j}\right), y\right]=\left(\left[x_{i j}, y\right]\right), y \in Y$, where $M_{\ell}(X)$ is the algebra of $\ell \times \ell$ matrices over $X$. Define

$$
\left(\operatorname{tr} \sharp \xi_{n}\right)\left(x^{0}, \ldots, x^{n} ; y^{0}, \ldots, y^{n-1}\right)=(\operatorname{tr} \sharp \tau)\left(x^{0}\left[x^{1}, y^{0}\right] \cdots\left[x^{n}, y^{n-1}\right]\right),
$$

for $x^{j} \in M_{\ell}(X), n \geq p$. For odd $n \geq p-1$ and $e \in \operatorname{Proj}\left(M_{\ell}(X)\right)$, let

$$
\operatorname{ch}_{e, n}\left(y^{0}, \ldots, y^{n}\right)=\frac{n !}{\left(\frac{n+1}{2}\right) !}(-2 \pi i)^{\frac{n+1}{2}} A_{y}\left(\operatorname{tr} \sharp \xi_{n+1}\right)\left(e, \ldots, e ; y^{0}, \ldots, y^{n}\right) .
$$

For $f \in Z_{\lambda}^{n}$, let $[f]=f+b C_{\lambda}^{n-1} \in H_{\lambda}^{n}=Z_{\lambda}^{n} / b C_{\lambda}^{n-1}$. Let $H_{\lambda}^{\text {odd }}(Y)$ be the group defined in [1].

Theorem 2. The mapping $[e] \mapsto\left[\mathrm{ch}_{e, 2 m-1}\right]$ is a homomorphism from $K_{0}(X)$ to $H_{\lambda}^{2 m-1}(Y), 2 m \geq p$. It satisfies

$$
\left[S \mathrm{ch}_{e, 2 m-1}\right]=\left[\mathrm{ch}_{e, 2 m+1}\right],
$$

and it defines a homomorphism from $K_{0}(X)$ to $H_{\lambda}^{\text {odd }}(Y)$. 
Proof. We only have to consider the case $e \in \operatorname{Proj}(X)$. It is easy to see (cf. [1]) that for even $n$,

$$
(b f)(e, \ldots, e)=f(e, \ldots, e)=0, \quad f \in C_{\lambda}^{n-1},
$$

and

$$
(A \hat{S} f)(e, \ldots, e)=(n+3)(n+2) f(e, \ldots, e) / 2, \quad f \in C^{n} .
$$

By $(7), b_{y} A_{y} \xi_{n}(e, \ldots, e ; y)=A_{y} \phi_{n}(e, \ldots, e ; y)=A_{y} \tau\left[e, y^{0}\right] \cdots\left[e, y^{n}\right]=0$ for odd $n \geq p-1$. Thus $\mathrm{ch}_{e, n} \in Z_{\lambda}^{n}(Y)$. On the other hand, $A_{x} \xi_{n}(e, \ldots, e ; y)=$ $(n+1) \xi_{n}(e, \ldots, e ; y)$ for even $n$. From (8), (9), (20) and (21), we have

$$
\begin{aligned}
& A_{y} \xi_{n}\left(e, \ldots, e ; y^{0}, \ldots, y^{n-1}\right)=b_{y}\left(A_{x} \hat{S}_{x} \theta_{n-2}\right)\left(e, \ldots, e ; y^{0}, \ldots, y^{n-1}\right) \\
& +(-1)^{m} \frac{(k-1) !}{(n-1) ! 2^{m}}\left[\left(A_{y} \hat{S}_{y}\right)^{m} A_{y} \xi_{k}\right]\left(e, \ldots, e ; y^{0}, \ldots, y^{n-1}\right),
\end{aligned}
$$

for $n=2 m+k$ and $k \geq p$, which proves (18). The rest of the proof is similar to the proof of Proposition 14 of Chapter II of [1].

Remark. By (12) and (21), for odd $n \geq p-1$, we also have

$$
\operatorname{ch}_{e, n}\left(y^{0}, \ldots, y^{n}\right)=\frac{n !(n+2)}{\left(\frac{n+1}{2}\right) ! 2}(-2 \pi i)^{\frac{n+1}{2}}\left(A_{y} \operatorname{tr} \sharp \eta_{n}\right)\left(e, \ldots, e ; y^{0}, \ldots, y^{n}\right) .
$$

For $\left(y_{i j}\right) \in M_{\ell}(Y)$, let $\left[x,\left(y_{i j}\right)\right]=\left(\left[x, y_{i j}\right]\right), x \in X$. Define $\operatorname{tr} \sharp \xi_{n}$ by (18) for $y^{j} \in M_{\ell}(Y)$ and $n \geq p$. For $u \in G L_{\ell}(Y)$ and even $n \geq p$, define

$$
\operatorname{ch}_{u, n}\left(x^{0}, \ldots, x^{n}\right)=k_{n}\left(A_{x} A_{y} \operatorname{tr} \sharp \xi_{n}\right)\left(x^{0}, \ldots, x^{n} ; u^{-1}, u, \ldots, u^{-1}, u\right),
$$

where $k_{n}=(-2 \pi i)^{n / 2} n ! /(n+1)\left(\frac{n}{2}\right) ! 2^{\frac{n}{2}}$.

Theorem 3. The mapping $[u] \mapsto\left[\mathrm{ch}_{u, 2 m}\right]$ is a homomorphism from $K_{1}(Y)$ to $H_{\lambda}^{2 m}(X)$, for $2 m \geq p$. It satisfies

$$
\left[S \operatorname{ch}_{u, 2 m}\right]=\left[\operatorname{ch}_{u, 2(m+1)}\right]
$$

and it defines a homomorphism from $K_{1}(Y)$ to $H_{\lambda}^{\text {even }}(X)$.

Proof. We only have to consider the case that $u \in Y$ and $u^{-1} \in Y$. It is easy to see that

$$
\left(A \phi_{2 m-1}\right)\left(x^{0}, \ldots, x^{2 m-1} ; u^{-1}, u, \ldots, u^{-1}, u\right)=0 .
$$

For $f \in C^{n}, n=2 m$,

$$
b A f=A b f-b f+\sum_{j=1}^{n}(-1)^{j-1}\left(f-t^{j} f\right)\left(a^{j} a^{j+1}, \ldots, a^{n+1}, a^{0}, \ldots\right) .
$$

By (4) and (7) we have

$$
\left(1-t_{x}\right) \xi_{n}=\left(1-t_{x}\right) b_{x} \psi_{n-1}=b_{x}^{\prime}\left(1-t_{x}\right) \psi_{n-1}=b_{x}^{\prime} \phi_{n-1}, \quad n \geq p+1 .
$$

From (24), it follows that $\left(1-t_{x}\right) A_{y} \xi_{2 m}\left(x^{0}, \ldots, x^{n} ; u^{-1}, u, \ldots, u^{-1}, u\right)=0$. By $b_{x} \xi_{n}=0$ and $(25)$, we have

$$
\left(b_{x} A_{x} A_{y} \xi_{2 m}\right)\left(x^{0}, \ldots, x^{2 m+1} ; u^{-1}, u, \ldots, u^{-1}, u\right)=0 .
$$


Thus $\mathrm{ch}_{u, 2 m} \in Z_{\lambda}^{2 m}$. Formula (23) comes from Theorem 1. Let us follow the lines of the proof of Proposition 15 of Chapter II of [1]. It is easy to see that

$$
\xi_{n}\left(x^{0}, \ldots, x^{n} ; y^{0}, \ldots, y^{n-1}\right)=0
$$

if one of the $y$ 's is 1 . Thus in $(22), u^{-1}$ and $u$ may be replaced by $u^{-1}-1$ and $u-1$ respectively.

For $f \in C_{\lambda}^{2 m-1}$, it is easy to calculate that

$$
(A \hat{S} f)\left(u^{-1}, u, \ldots, u^{-1}, u\right)=4(m+1) m f\left(u^{-1}, u, \ldots, u^{-1}, u\right) .
$$

Similar to the proof of Proposition 15 of Chapter II of [1], we may prove that $\operatorname{ch}_{u v, 2 m}-\operatorname{ch}_{u, 2 m}-\operatorname{ch}_{v, 2 m}$ is the boundary of a cyclic cochain in $C_{\lambda}^{2 m-1}(X)$ since

$$
b_{y} A_{y} \xi_{n}=A \phi_{n}=-b_{x} A_{x} \eta_{n}
$$

by (7), which proves the theorem.

Remark. Similar to (15), by (6), we have $A_{x} \eta_{m}=m \eta_{m}+t_{x} p_{x} b_{y} \phi_{m-1}$. Suppose $n$ is even and $\geq p+1$. By (24), we have

$$
\begin{aligned}
\left(A_{x} \eta_{n-1}\right)\left(x^{0}, \ldots, x^{n-2} ; u^{-1}-1, u-1, \ldots, u^{-1}-1, u-1\right) & \\
& =(n-1) \eta_{n-1}\left(x^{0}, \ldots, x^{n-2} ; u^{-1}-1, u-1, \ldots, u^{-1}-1, u-1\right) .
\end{aligned}
$$

By (12), we may prove that

$$
\left(A_{x} A_{y} \xi_{n}-\frac{(n+1)}{n(n-1)} \hat{S}_{x} A_{x} A_{y} \eta_{n-1}\right)\left(\cdot ; u^{-1}-1, \ldots, u-1\right) \in b C_{\lambda}^{n-1}(X) .
$$

Thus for even $n \geq p-1$, we may define

$$
\begin{aligned}
\operatorname{ch}_{u, n} & \left(x^{0}, \ldots, x^{n}\right) \\
& =\hat{k}_{n}\left(A_{x} A_{y} \operatorname{tr} \sharp \eta_{n+1}\right)\left(x^{0}, \ldots, x^{n} ; u^{-1}-1, u-1, \ldots, u^{-1}-1, u-1\right),
\end{aligned}
$$

where $\hat{k}_{n}=-(-2 \pi i)^{n / 2} n ! /\left(\frac{n+2}{2}\right) ! 2^{\frac{n+2}{2}}$.

Now let us consider the case that $X$ and $Y$ are $p$-almost commuting subalgebras of the operator algebra on a complex separable Hilbert space $\mathcal{H}$ and $\tau$ is the usual trace. In this case, the elements of $M_{\ell}(X)$ or $M_{\ell}(Y)$ may be regarded as operators on $\mathcal{H} \otimes \mathbb{C}^{\ell}$.

Theorem 4. The index map

$$
\begin{aligned}
\langle[e],[u]\rangle & =\frac{1}{m}(-1)^{m}\left(A_{y} \operatorname{tr} \sharp \xi_{2 m}\right)\left(e, \ldots, e ; u^{-1}, u, \ldots, u^{-1}, u\right) \\
& =\left.\operatorname{index~} e u\right|_{\operatorname{range}(e)},
\end{aligned}
$$

where $e \in \operatorname{Proj} M_{\ell}(X)$ and $u \in G L_{\ell}(Y)$, is a homomorphism from $K_{0}(X) \times K_{1}(Y)$ to $\mathbb{Z}$.

Proof. It is easy to calculate that

$$
\langle[e],[u]\rangle=\operatorname{trace}\left((I-c a)^{m}-(I-a c)^{m}\right),
$$

where $a=\left.e u\right|_{\text {range(e) }}$ and $c=\left.e u^{-1}\right|_{\text {range(e) }}$. The rest of the proof is similar to the corresponding part of Chapter I of [1].

Example. Suppose $e$ is the orthogonal projection from $L^{2}(\mathbb{T})$ to $H^{2}(\mathbb{T})$, and $u$ is the backward bilateral shift $(u f)(z)=\bar{z} f(z)$. Then $\langle[e],[u]\rangle=1$. 


\section{Deformed COMmutator CASE}

Let $\mathcal{A}$ be an algebra over $\mathbb{C}$ with trace ideal $J$ and trace $\tau$. Let $X$ and $Y$ be subgroups of $\mathcal{A}$. Assume that there is a function $q: X \times Y \rightarrow \mathbb{C}$ satisfying

$$
q\left(x^{1} x^{2} ; y^{1} y^{2}\right)=\prod_{i, j=1}^{2} q\left(x^{i}, y^{j}\right) \quad \text { and } \quad q(x, 1)=q(1, y)=1
$$

for $x^{i}, x \in X$ and $y^{j}, y \in Y$. Let $\{x, y\}=x y-q(x, y) y x$ be the $q$-deformed commutator. Assume that there is a natural number $p$ such that

$$
\left\{x^{1}, y^{1}\right\} \cdots\left\{x^{p}, y^{p}\right\} \in J
$$

(We say that $X$ and $Y$ are $p$-almost $q$-deformed commuting.) Let

$$
M^{m, n}=\left\{\left(x^{0}, \ldots, x^{m} ; y^{0}, \ldots, y^{n}\right) \in X^{m+1} \times Y^{n+1}: q\left(x^{0} \cdots x^{m}, y^{0} \cdots y^{n}\right)=1\right\} .
$$

By means of the operation defined in [9], we may generalize Theorem 1 to the following.

Theorem $\mathbf{1}^{\prime}$. Let $k$ be an even number, $k \geq p, m \in \mathbb{N}$, and $n=k+2 m$. Then there exists $\theta_{j}=\theta_{j}\left(x^{0}, \ldots, x^{j} ; y^{0}, \ldots, y^{j}\right)$ satisfying $\tau_{x} \theta_{j}=\tau_{y} \theta_{j}$ on $M^{j, j}$ for $j=n-1$ and $n-2$ such that

$$
\alpha_{x} \alpha_{y} \xi_{n}=\delta_{x} \theta_{n-1}-\alpha_{x} S_{x} \delta_{y} \theta_{n-2}+\tilde{\xi}_{n} \quad \text { on } M^{n, n-1},
$$

where

$$
\tilde{\xi}_{n}=\frac{1}{(2 \pi)^{2 m}} \frac{k !(k-1) !}{n !(n-1) !}\left(\alpha_{x} S_{x}\right)^{m}\left(\alpha_{y} S_{y}\right)^{m} \alpha_{x} \alpha_{y} \xi_{k} .
$$

Let $Q=\{q(x, y): x \in X, y \in Y\}$. Let $\mathcal{W}=\{(x, y, c): x \in X, y \in Y, c \in Q\}$ be the group with multiplication $\left(x^{0}, y^{0}, c^{0}\right)\left(x^{1}, y^{1}, c^{1}\right)=\left(x^{0} x^{1}, y^{0} y^{1}, c^{0} c^{1} q\left(x^{0}, y^{1}\right)\right)$. Let $p: \mathcal{W} \rightarrow \mathcal{A}$ be the mapping $p(x, y, c)=$ cyx. Then the "curvature" of this mapping is defined as $\omega\left(w^{0}, w^{1}\right)=p\left(w^{0} w^{1}\right)-p\left(w^{0}\right) p\left(w^{1}\right), w^{0}, w^{1} \in \mathcal{W}$. For $n \geq p$, define the Chern character of dimension $2 n-1$ (see [3] and [5]) as

$$
\begin{aligned}
& \operatorname{ch}_{2 n-1}\left(w^{0}, \ldots, w^{2 n-1}\right) \\
& \quad=\tau\left(\omega\left(w^{0}, w^{1}\right) \cdots \omega\left(w^{2 n-2}, w^{2 n-1}\right)-\omega\left(w^{2 n-1}, w^{0}\right) \cdots \omega\left(w^{2 n-3}, w^{2 n-2}\right)\right) .
\end{aligned}
$$

Theorem 5. If $p=1$ or 2 , then there are $(2 n-2)$-cyclic cochains $f_{2 n-2}$ such that

$$
\mathrm{ch}_{2 n-1}=b f_{2 n-2} \quad \text { off } M^{2 n-1,2 n-1}
$$

for $n \geq p$.

Proof. By the proof of the corollary of Theorem 2 in [9], it is easy to see that (26) holds for $n=p$. Then the formula (26) for $n>p$ follows from the fact that there is a constant $k_{n}$ such that $\mathrm{ch}_{2 n+1}$ and $k_{n} S \mathrm{ch}_{2 n-1}$ are in the same cohomology class by Proposition 15 of Chapter II of [1].

\section{REFERENCES}

1. A. Connes, Non-commutative differential geometry, Inst. Hautes Études Sci. Publ. Math. 62 (1985), 257-360. MR 87i:58162

2. , Noncommutative Geometry, Acad. Press, Inc., San Diego, 1994. MR 95j:46063

3. J. Cuntz, Cyclic cohomology and K-homology, Proc. Inter. Congr. Math. (Kyoto, 1990), Math. Soc. Jap., 1991, pp. 968-978. MR 93c:19003 
4. J. L. Loday and D. Quillen, Cyclic homology and the Lie algebra homology of matrices, Comment. Math. Helvetici 59 (1984), 565-591. MR 86i:17003

5. D. Quillen, Algebra cochains and cyclic cohomology, Publ. Math. IHES 68 (1989), 139-174. MR 90j: 18008

6. D. Xia, Trace formula for almost Lie group of operators and cyclic one-cocycles, Integr. Equat. Oper. Th. 9 (1986), 570-587. MR 88d:22015

7. _ Trace formulas for almost commuting operators, cyclic cohomology and subnormal operators, Integr. Equat. Oper. Th. 14 (1991), 276-298. MR 92a:47046

8. , A note on the trace formulas for almost commuting operators, Proc. Amer. Math. Soc. 116 (1992), 135-141. MR 92k:47074

9. Generalized cyclic cohomology associated with deformed commutators, Proc. Amer. Math. Soc. 124 (1996), 1743-1753.

Department of Mathematics, Vanderbilt University, Nashville, Tennessee 37240

E-mail address: xiad@ctrvax.vanderbilt.edu 